\title{
Electron scattering in large water clusters from photoelectron imaging with high harmonic radiation
}

\section{Journal Article}

Author(s):

Gartmann, Thomas E.; Hartweg, Sebastian; Ban, Loren (1); Chasovskikh, Egor; Yoder, Bruce L.; Signorell, Ruth (1)

Publication date:

2018-06

Permanent link:

https://doi.org/10.3929/ethz-b-000274702

Rights / license:

In Copyright - Non-Commercial Use Permitted

Originally published in:

Physical Chemistry Chemical Physics 20(24), https://doi.org/10.1039/c8cp02148a

\section{Funding acknowledgement:}

172472 - Phase Transitions of Ultrafine Aerosol Particles: Condensation, Freezing, and Metal Formation in Confined Systems (SNF) 


\section{PCCP}

\section{PAPER}

\section{Electron scattering in large water clusters from photoelectron imaging with high harmonic radiation}

Received 00th January 20xx, Accepted 00th January 20xx

DOI: $10.1039 / x 0 \times x 00000 x$

www.rsc.org/

\section{Thomas E. Gartmann¥, Sebastian Hartweg‡, Loren Ban, Egor Chasovskikh, Bruce L. Yoder and Ruth Signorell}

\begin{abstract}
Low-energy electron scattering in water clusters $\left(\mathrm{H}_{2} \mathrm{O}\right)_{n}$ with average cluster sizes of $\langle n\rangle<700$ is investigated by angleresolved photoelectron spectroscopy using high harmonic radiation at photon energies of 14.0, 20.3, and 26.5eV for ionization from the three outermost valence orbitals. The measurements probe the evolution of the photoelectron anisotropy parameter $\beta$ as a function of cluster size. A remarkably steep decrease of $\beta$ with increasing cluster size is observed, which for the largest clusters reaches liquid bulk values. Detailed electron scattering calculations reveal that neither gas nor condensed phase scattering can explain the cluster data. Qualitative agreement between experiment and simulations is obtained with scattering calculations that treat cluster scattering as an intermediate case between gas and condensed phase scattering.
\end{abstract}

\section{Introduction}

It has been shown that the transport properties of electrons in water play a significant role in various processes ranging from radiation damage and atmospheric aerosol chemistry to liquid phase photoelectron spectroscopy. 1-8 Despite numerous experimental and theoretical efforts (see refs. ${ }^{4-6,8-20}$ and references therein), a comprehensive understanding of the transport scattering of low-energy electrons $(<100 \mathrm{eV})$ in liquid water has still not been fully reached. Over the kinetic energy (eKE) range from 1.7 to $100 \mathrm{eV}$, detailed scattering data (differential scattering cross sections and energy losses) for all important scattering channels (elastic, phonon, vibron, and electronic) are only available for amorphous ice from highresolution electron-energy-loss studies by Sanche and coworkers, ${ }^{12,13}$ but not for liquid water. Thus, for lack of liquid water data these ice data and/or rescaled gas phase data ${ }^{21}$ have often been used in scattering simulations - the error inherent in this approach was unknown. The first scattering data for the liquid state for eKE $\lesssim 6 \mathrm{eV}$ were recently retrieved in our research group from photoelectron velocity-map imaging (VMI) studies of water droplets. ${ }^{7,} 17$ This study also revealed that for eKEs between $1.7 \mathrm{eV}$ and $\sim 6 \mathrm{eV}$ the scattering parameters of liquid water and amorphous ice are virtually indistinguishable within current experimental uncertainties, confirming a previous expectation expressed by

Department of Chemistry and Applied Biosciences, Laboratory of Physical Chemistry, ETH Zürich, Vladimir-Prelog-Weg 2, CH-8093 Zürich, Switzerland Email: rsignorell@ethz.ch

+ Electronic Supplementary Information (ESI) available:. See DOI: $10.1039 / x 0 x x 00000 x$

$\ddagger$ These authors contributed equally to this work.
Sanche and coworkers. ${ }^{13}$ Similarly, a recent approach that combines data from small water clusters $\left(\left(\mathrm{H}_{2} \mathrm{O}\right)_{n}\right.$ with $\left.n \leq 20\right)$, water droplets, and a liquid water microjet with a detailed scattering model ${ }^{17,20,22}$ provides first hints that this similarity between liquid and ice extends up to eKEs of $\sim 20 \mathrm{eV}-$ at least for the elastic, phonon, and vibron cross sections.

The aim of the present work is to clarify whether condensed phase scattering data (liquid/amorphous ice ${ }^{7,12,13,17,20}$ ) are also suitable for the description of electron transport scattering ${ }^{20}$ for eKEs below $\sim 20 \mathrm{eV}$ in large, neutral water clusters $\left(\mathrm{H}_{2} \mathrm{O}\right)_{n}$ with $\mathrm{n}<$ 2500. We focus on the influence of scattering on the photoelectron anisotropy (PA) for ionization from the three valence orbitals $1 b_{1}$, $3 a_{1}$, and $1 b_{2}$ using high harmonic radiation at photon energies of $h v=14.0,20.3$, and $26.5 \mathrm{eV}$. The PA is described by a single anisotropy parameter $\beta$, defined by

$$
I(\theta) \propto 1+\frac{\beta}{2}\left(3 \cos ^{2} \theta-1\right) .
$$

$\theta$ is the angle between the light polarization and the ejection direction of the photoelectrons. $I(\theta)$ is the electron signal detected at that angle. $\beta$ is mainly sensitive to elastic, phonon, and vibron scattering, which in the eKE range studied are similar for the liquid and for amorphous ice (see above).

Previous valence photoelectron spectra of neutral water clusters have revealed size-dependent shifts of band positions of up to $\sim 1 \mathrm{eV}$ toward lower values compared with the monomer, accompanied by band broadenings. ${ }^{20,} 23-25$ Both trends were mainly attributed to condensation, neglecting contributions from scattering. The influence of electron scattering was only discussed in connection with the PA for clusters with less than $\sim 80$ water molecules. ${ }^{18,} 20$ In our recent photoelectron photoion coincidence study of water clusters, ${ }^{20}$ we have proposed that two distinct scattering processes 
explain electron scattering in the liquid bulk. The first process is the photoionization itself (referred to as "genuine scattering"); i. e. the formation of an unbound electron in the conduction band, while the second process describes the transport scattering of the unbound electron through the water matrix before it is ejected into vacuum (referred to as "transport scattering"). Recent work on liquid microjets has revealed that this two-step model is indeed capable of providing the first quantitative description of electron scattering in liquid water. ${ }^{7,22}$ The current investigation was intended to bridge the gap between these previous small cluster and the condensed phase studies.

The number of size-dependent studies of the photoelectron angular distribution (PAD) - and thus the information on electron scattering in clusters - is very limited even for other neutral molecular and atomic (rare-gas) clusters. ${ }^{26-34}$ For (larger) neutral clusters, size-selection is not possible and the accurate determination of cluster size distributions remains a major issue. To avoid unknown errors associated with scaling laws, ${ }^{35}$ we have recorded the cluster size distributions directly with the Na-doping method originally proposed by Buck and coworkers. ${ }^{36-40}$ We would also like to mention that even though a fair number of PAD studies on size-selected anion clusters of various sizes exist (see refs..$^{41-46}$ and refs. therein), we are not aware of any investigation on anion clusters that addresses the influence of transport scattering except for the modelling provided in ref. ${ }^{46}$.

\section{Experiment}

Size-dependent photoelectron images of water clusters were recorded with a velocity-map imaging (VMI) ${ }^{47,48}$ photoelectron spectrometer. ${ }^{26-29,} 49$ The spectrometer in Fig. 1a consists of three vacuum chambers for cluster formation ("inlet chamber"), cluster sizing ("Na-oven chamber"), and electron/ion detection ("detection chamber"). Water clusters were created by expanding water vapour through a pulsed $(500 \mathrm{~Hz})$ Even-Lavie (EL) valve ${ }^{50}$ into vacuum. Depending on the desired cluster size, the temperature of the sample tube with liquid water was varied between $333 \mathrm{~K}$ and $443 \mathrm{~K}$. To avoid condensation, the subsequent tubing and the $\mathrm{EL}$ valve were kept at temperatures that were higher by $5 \mathrm{~K}$ and $10 \mathrm{~K}$, respectively. Either pure water vapour or a mixture of water vapour with helium $(\mathrm{He})$ or neon $(\mathrm{Ne})$ carrier gas at pressures between 1.6bar and 13bar was expanded. Identical results were found for pure water and the mixtures.

For photoelectron imaging, the Na-oven chamber served only as a differential pumping chamber. Photoelectrons were produced between the repeller (R) and extractor (E) plates of the VMI optics by single-photon ionization with extreme ultraviolet (EUV) light from a home-built EUV laser based on High Harmonic Generation (HHG) (Fig. 1b; see below). VMI photoelectron images were recorded at a rate of $500 \mathrm{~Hz}$ with a multichannel plates/phosphor screen assembly (MCP/PS) and a CMOS camera (Fig. 1a). The images were subsequently centroided and summed. Background images containing water
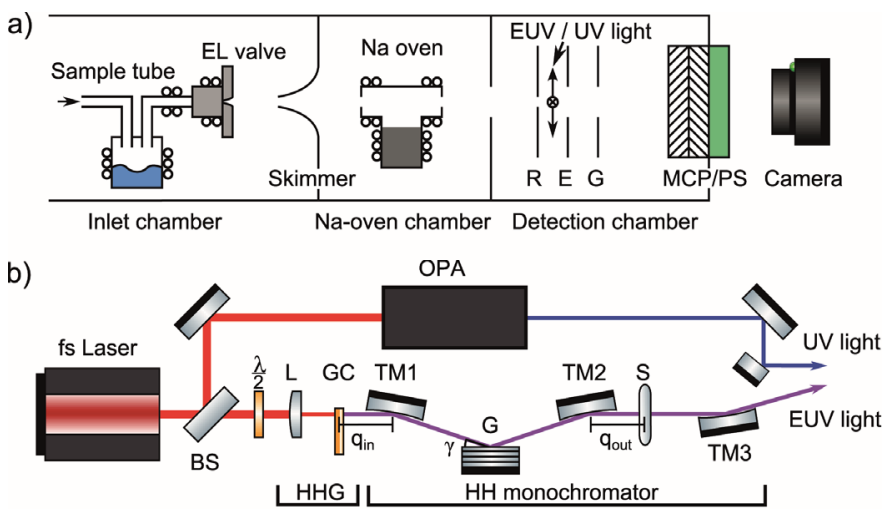

Fig. 1 Scheme of the experimental setup. a) Cluster source, Naoven chamber, and photoelectron/mass spectrometer. b) UV and EUV laser light source with high harmonic generation (HHG) and $\mathrm{HH}$ monochromator ${ }^{51,52}$. BS: beam splitter, $\lambda / 2$ : half-wave plate, L: focusing lens, GC: gas cell, TM1, TM2 and TM3: toroidal mirrors, $\mathrm{q}_{\mathrm{in}} / \mathrm{q}_{\text {out }}$ : input/output arms, G: grating, $\gamma:$ altitude angle, $\mathrm{s}$ : exit slit.

monomer only (without water clusters) were recorded and subtracted from the cluster photoelectron images to remove contributions from the monomer. Photoelectron images, photoelectron spectra, and anisotropy parameters $\beta$ were recovered from the background-subtracted data using the pBASEX image inversion algorithm ${ }^{53}$ (see example image and spectrum in Figs. $2 a$ and $b$ ). The band position of the water monomer ( $1 b_{1}$ band) was used for energy calibration.

Photoemission experiments were performed at three different photon energies $h v=14.0,20.3$, and $26.5 \mathrm{eV}\left(9^{\text {th }}\right.$, $13^{\text {th }}$, and $17^{\text {th }}$ harmonic, respectively). High-order laser harmonics $(\mathrm{HHs} ;)^{54}$ were generated by focusing a $1 \mathrm{kHz}, 35 \mathrm{fs}$ Ti:Sapphire laser at a wavelength of $795 \mathrm{~nm}$ (Coherent, Astrella) in an argon filled gas cell (GC) (Fig. 1b). A home-built time-preserving monochromator in the off-plane mount geometry following the design by Poletto and coworkers ${ }^{51}, 52$ was used for the spectral selection of the HHs. Depending on the operation parameters, the monochromator provides photon fluxes between $10^{8}$ and $10^{11}$ photons/s at the output with EUV pulse durations between $50 \mathrm{fs}$ and $80 \mathrm{fs}^{55}$.

The cluster size distributions were determined in situ, but independently from the photoelectron images, by the $\mathrm{Na}$ doping method before and/or after the photoemission experiments. The Na-doping method is described in detail in refs. ${ }^{26,36-40}$ It provides a direct and accurate measurement of the size distribution for neutral weakly-bound clusters. Briefly, the water clusters were doped with $\mathrm{Na}$ atoms as they traversed the hot Na oven (Fig. 1a). The $\mathrm{Na} 3$ s electrons of the $\mathrm{Na}$-doped clusters were then ionized in the detection chamber with $280 \mathrm{~nm}$ UV light provided by an optical parametric amplifier (OPA, Coherent, OPerA Solo), which was pumped by the Ti:Sapphire laser (Fig. 1b). The cluster mass distributions were then recorded by time-of-flight mass spectrometry. High extraction voltages ensured quantitative detection also for large cluster masses ( $n \approx 1500-2000$ ). Conversion of the cluster mass to the number of water molecules $n$ per cluster provides 
cluster size distributions (Fig. 2c). As described in section 1 in the ESIt, this conversion takes into account the different sizedependence of the ionization cross sections of the Na-doping method and EUV photoionization. For simplicity, we use the average cluster size $\langle n\rangle$ throughout this paper, defined by

$$
\langle n\rangle=\frac{\sum_{i}^{n_{\max }} w_{i} n_{i}}{\sum_{i}^{n_{\max }} w_{i}} .
$$

The weight $w_{i}$ is the relative abundance of clusters with $n_{i}$ water molecules. For the scattering simulations, we have tested that a simulation for the average cluster size $\langle n\rangle$ provides very similar results as the explicit simulation for the whole size distribution.
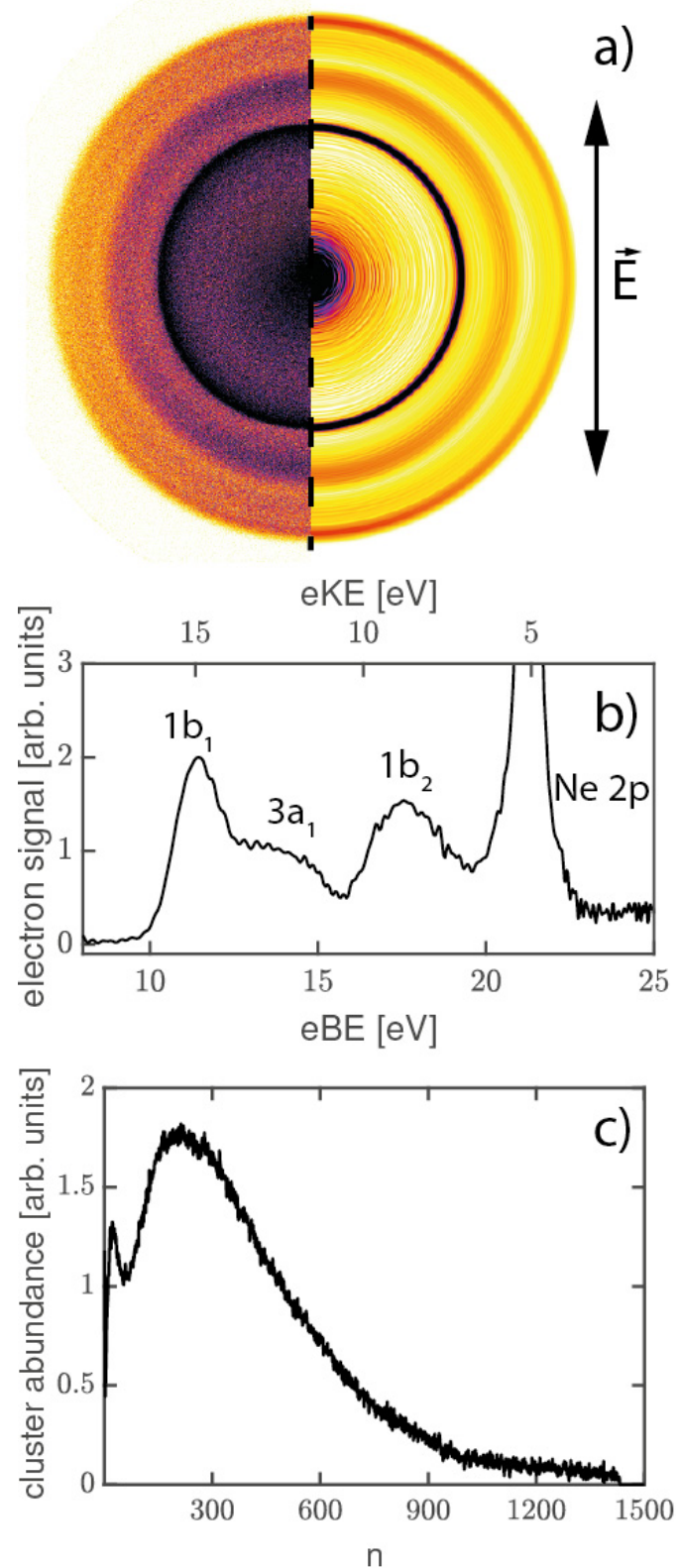

Fig. 2 a) Example photoelectron image for water clusters seeded in Ne gas with an average cluster size of $\langle n\rangle=361$ after ionization with photons of $h v=26.5 \mathrm{eV}$. Left: raw image, Right: reconstructed image. $\vec{E}$ indicates the polarization direction of the light. Black is high and yellow is low electron intensity. b) Corresponding photoelectron spectrum in the valence region of the water clusters. c) Corresponding cluster size distribution recorded with the $\mathrm{Na}$ doping method, after corrections (ESI + ).

\section{Scattering simulations}

For details of the electron scattering simulations, we refer the reader to the Supporting Information of refs. ${ }^{7,17,20}$. Briefly, we used a probabilistic electron scattering model based on a Monte Carlo solution of the transport problem. The simulations explicitly take into account the scattering cross sections, their angular dependences, and the energy loss functions of all relevant scattering processes; i.e. elastic scattering and inelastic phonon, vibron, and electronic scattering. To understand the experimental cluster data, we used four different sets of scattering parameters (i)-(iv) as input for the calculations, which are described in the following. The scattering cross sections for the four cases (i)-(iv) are shown in section 2 in the ESIt together with additional descriptions.

(i) This parameter set represents transport scattering in clusters as in the condensed bulk phase (liquid/amorphous ice). We used the scattering parameters derived in refs. ${ }^{7,}{ }^{17}$ and ref. ${ }^{13}$ for eKEs below and above $\sim 6 \mathrm{eV}$, respectively.

(ii) The other extreme is electron scattering as in the gas phase. This case was represented by up-scaling the condensed phase cross sections ${ }^{7}, 13,17$ to match the higher gas phase values from ref. ${ }^{21}$. The scaling was performed separately for the quasi-elastic (elastic and phonon), the vibron, and the electronic channels, resulting in scaling factors of $22,3.5$, and 3 , respectively.

(iii) The absence of dielectric screening in the gas phase is one factor that contributes to the higher cross sections in the gas compared with the condensed phase. To test the effect of the missing dielectric screening, we have upscaled all condensed phase cross sections $s^{7,13,17}$ by the square of the optical dielectric constant $\varepsilon^{2}=1.8^{2}=3.24$ 25 . This scaling factor is very similar to the scaling factors found for the vibron and electronic channels in case (ii), but differs substantially from case (ii) for the quasi-elastic channels.

(iv) Similarly, instead of up-scaling the condensed phase cross sections one can down-scale the gas phase cross sections from case (ii) by a factor of $1 / \varepsilon^{2}=1 / 1.8^{2}=0.31$. This corresponds to adding dielectric screening to the gas phase cross sections.

Cases (iii) and (iv) represent simple intermediate cases of electron scattering in between the condensed and the gas phase extreme. In case (iii), the quasi-elastic cross sections are closer to the condensed than to the gas phase cross sections, while the vibron and electronic cross sections are essentially 
the same as in the gas phase. The opposite holds for case (iv), where the quasi-elastic cross sections are closer to the gas phase cross sections and the vibron and electronic cross sections are essentially the same as in the condensed phase. The input values for the anisotropy parameters $\beta_{\text {inp }}$ were adjusted to reproduce the experimental $\beta$ value for small clusters $(n=6)$ from ref. ${ }^{20}$ - except in case (II) where this was not possible (see section 4.2).

\section{Results and discussion}

\subsection{Size-dependent electron binding energy}

Fig. 3 and Table S1 in the ESIt present a summary of the absolute shift $\triangle \mathrm{eBE}=|\mathrm{eBE}(\mathrm{c})-\mathrm{eBE}(\mathrm{m})|$ of the electron binding energy of the cluster, $\mathrm{eBE}(\mathrm{c})$, relative to that of the monomer, $\mathrm{eBE}(\mathrm{m}),{ }^{56}$ as a function of $\langle n\rangle^{-1 / 3}$ for the three orbitals $1 b_{1}, 3 a_{1}$, and $1 b_{2}$. Black dots indicate cluster data from the present work, green diamonds show data from ref. ${ }^{20}$, and red crosses summarize data from refs. ${ }^{18,}$ 23-25, 28, 57. Fig. 3 also includes eBE shifts for liquid water (closed blue triangles) and solid water (open blue triangles). ${ }^{56}$

The $\mathrm{eBE}(\mathrm{c})$ decreases continuously with increasing cluster size. For the largest clusters, $\triangle \mathrm{eBE}$ amounts up to $\sim 1 \mathrm{eV}$. Similar sizedependent shifts were also observed for ammonia and dimethyl ether clusters. ${ }^{26,27}$ Condensation of water is also accompanied by a substantial broadening of the band widths up to several times the values for the gas phase monomer (refs. ${ }^{23,24,56}$ and Table S1 in the ESI $)$. Note that a meaningful quantification of the broadening is difficult due to other experimental broadenings and potential band splittings.

Potential contributions (e.g. change in the initial state energy, final state polarization screening, electron scattering, surface charges) to the downward shift of the $\operatorname{eBE}(\mathrm{c})$ with increasing cluster size have previously been discussed. ${ }^{18,23-25,28,57}$ A conclusive explanation for binding energy shifts and band broadening in water clusters, however, has not yet been reached. Polarization screening models, which have been proven successful for the description of eBE(c) for core orbital ionization of rare gas clusters, ${ }^{58}$ are likely not sufficient to explain binding energy shifts for valence orbitals of strongly hydrogen-bonded clusters. The lack of simple models for the evolution of the $\mathrm{eBE}(\mathrm{c})$ of water clusters also renders the extrapolation from cluster data to "infinite" solid/liquid bulk questionable. For these reasons, we refrain from fitting polarization screening models to the cluster data and from extrapolating cluster data to bulk in Fig. 3. Even though a straightforward explanation for the evolution of binding energy shifts and band broadenings is not possible at this point, the comprehensive data set obtained in this work provides a useful reference for future in-depth modelling studies.
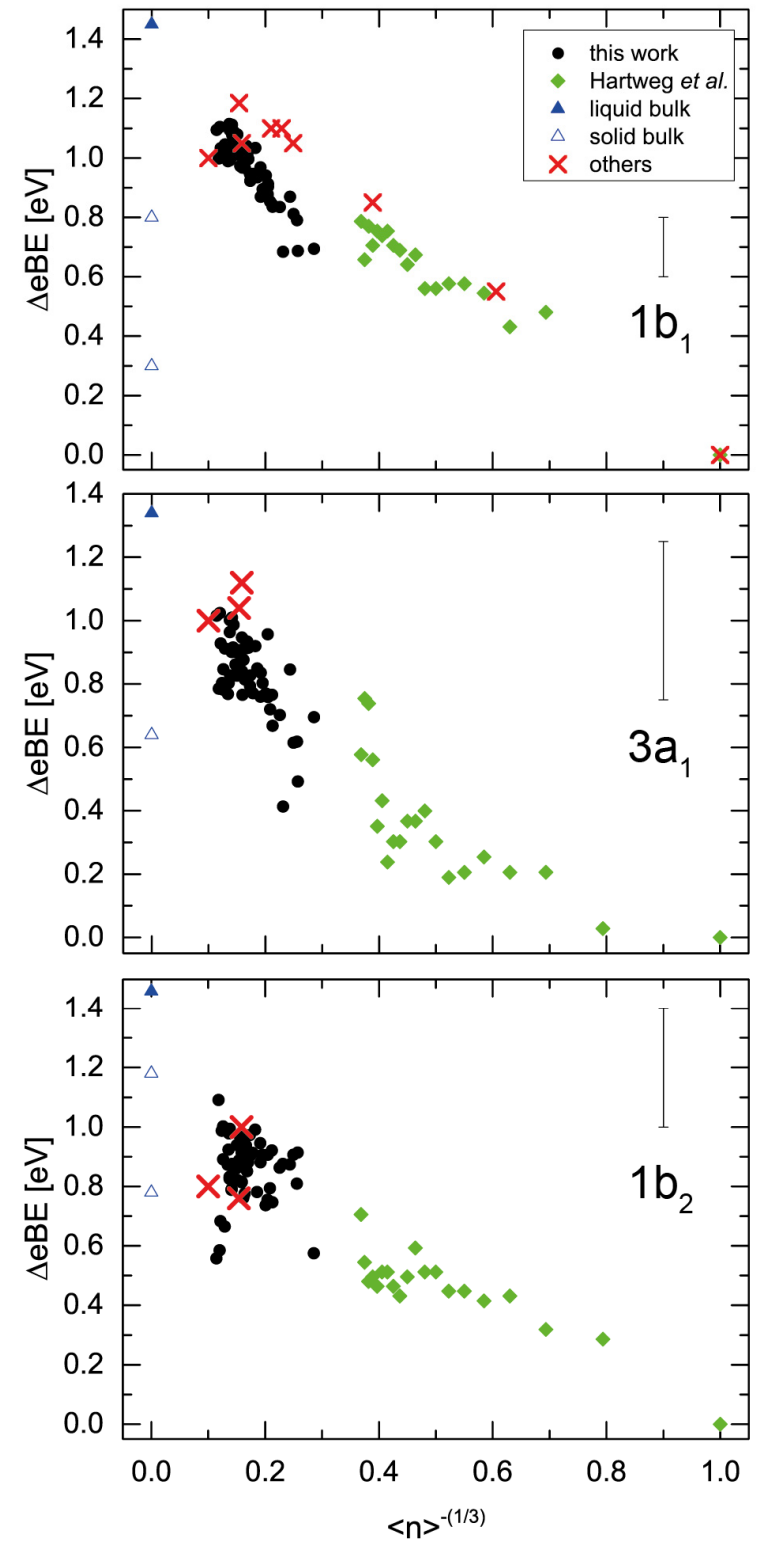

Fig. 3 Absolute shifts $\triangle \mathrm{eBE}$ of the electron binding energy of the clusters relative to that of the monomer as a function of $\langle n\rangle^{-1 / 3}$ for ionization from the $1 b_{1}$ (top), $3 a_{1}$ (middle), and $1 b_{2}$ (bottom) orbitals. Black dots: present work; Green diamonds: data from ref. ${ }^{20}$; Red crosses: data from refs. ${ }^{18,} 23-25,28,57$. Closed blue triangles: corresponding liquid water data ${ }^{56}$; Open blue triangles: corresponding solid water data (see refs. in ${ }^{56}$ ). Typical uncertainties of the absolute values of the present data are indicated by the error bars in each panel (see ESI $\dagger$ ).

\subsection{Size-dependent anisotropy parameter}

The black circles in Fig. 4 (Table S2 in the ESIt) show a systematic decrease of the experimental $\beta$ parameters with increasing cluster size $\langle n\rangle$ for photoionization from the $1 b_{1}$ orbital. The absolute decrease becomes smaller for ionization at lower photon energy $h v$ because the genuine PAD is already more isotropic at lower eKE. Similar trends were observed for ionization from the $3 a_{1}$, and $1 b_{2}$ orbital (Figs. S4 
and S5, respectively, and Table S2 in the ESIt). The largest absolute decrease is observed for the out-of-plane lone pair $\left(1 b_{1}\right.$; Fig. 4), which acts as the hydrogen bond acceptor, followed by the in-plane lone pair ( $3 \mathrm{a}_{1}$; Fig. S4 ESIt) and the $\sigma_{\mathrm{OH}}$ bond orbital $\left(1 \mathrm{~b}_{2}\right.$; Fig. S5 ESI + ). This trend reflects the decreasing genuine PA from the $1 b_{1}$ to the $1 b_{2}$ orbital. The evolution towards more isotropic PADs with increasing cluster size agrees with the trend we previously observed for very small water clusters $(n<20)^{20}$. The present study extends these previous data to substantially larger clusters. We are aware of only one other study by Zhang et al. that reports $\beta$ parameters for water clusters with $\langle n\rangle=58$ and $84 .{ }^{18} \mathrm{~A}$ direct comparison with the present cluster data is, however, not possible because those measurements were performed at higher photon energies $h v=40$ and $60 \mathrm{eV}$.

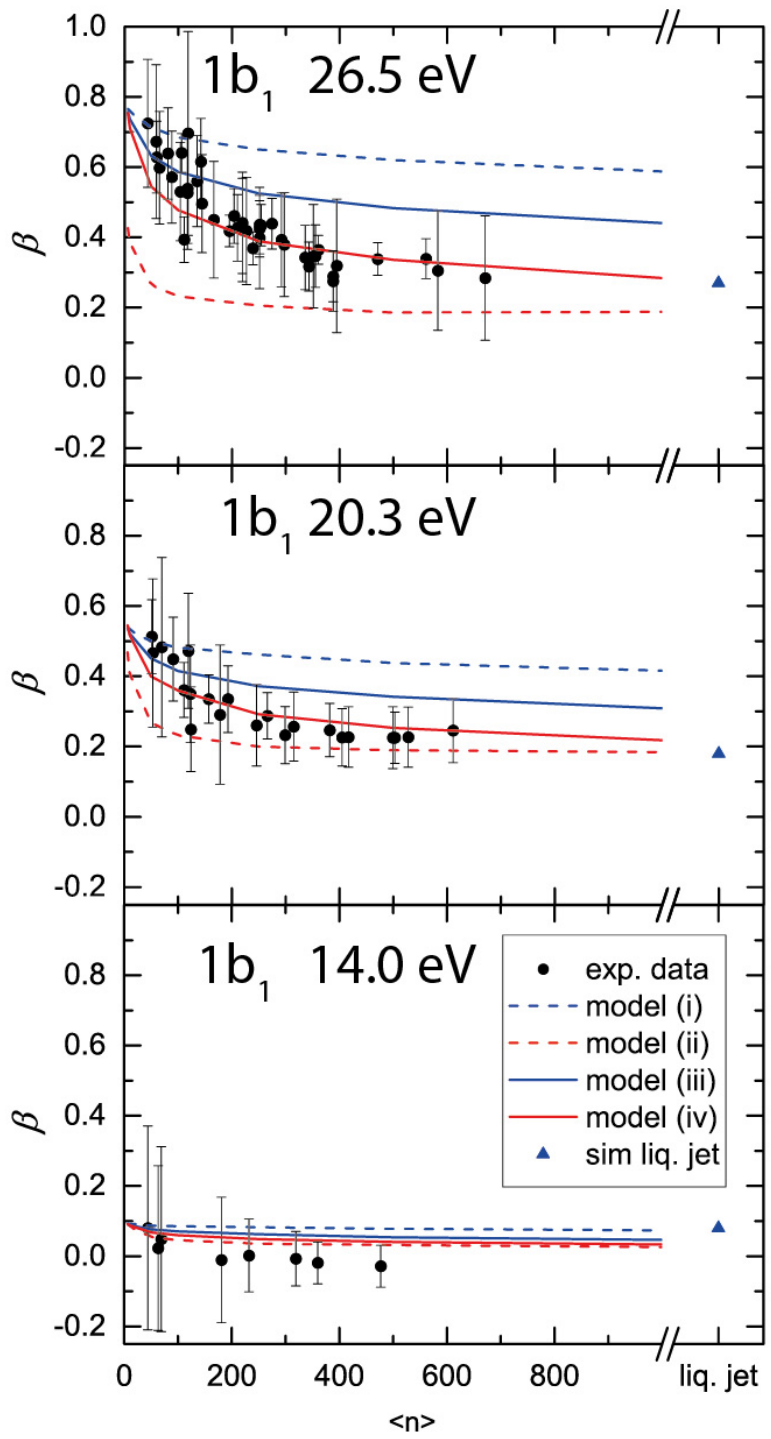

Fig. $4 \beta$ parameters as a function of cluster size $\langle n\rangle$ for photoionization from the $1 \mathrm{~b}_{1}$ orbital using $\mathrm{HH}$ radiation of $h \mathrm{v}$ $=26.5 \mathrm{eV}$ (top), $20.3 \mathrm{eV}$ (middle), and 14.0eV (bottom). Black circles: experimental data. Dashed blue lines: simulation with model (i). Dashed red lines: simulation with model (ii). Full blue lines: simulation with model (iii). Full red lines: simulation with model (iv). Blue triangles: prediction for liquid jet (corrected for the polarization dependent coupling of the laser light into the jet, see $\mathrm{ESI}+$ ) using the scattering parameters from model (i).

The anisotropy parameters of the largest clusters in Fig. 4 already reach values close to those of the liquid bulk (see blue triangle for liquid microjet values and refs. ${ }^{20,}{ }^{22}$ ). This rather surprising result already indicates that condensed phase scattering data (case (i) in section 3) are not suitable for describing transport scattering in these large clusters. Simulation (i) in Fig. 4 (dashed blue lines) confirms this interpretation. The condensed phase scattering cross sections are too small to reproduce the experimentally observed decrease in the PA. The deviation is particularly pronounced for the larger clusters for which one would naively have expected the condensed phase description to perform better than for smaller clusters. Using the gas phase description of scattering (case (ii); dashed red lines), we find almost the opposite behaviour. While the condensed phase cross sections are too small to reproduce the experimental data, the gas phase cross sections are too high resulting in PADs that are generally too isotropic compared with those we observe experimentally. For the gas phase scattering simulations (ii) we would have had to assume unreasonable $\beta_{\text {inp }}$ values exceeding the monomer values in order to match the experimental anisotropy parameters for very small clusters $(n=6) .{ }^{20}$ In the case of excitation with the $17^{\text {th }}$ harmonic, $\beta_{\text {inp }}$ would even have exceeded the physical maximum of 2 . For the simulation in Fig. 4, we have instead chosen more reasonable $\beta_{\text {inp }}$ not exceeding the monomer values. These are the highest physically justifiable values, which, however, can obviously not reproduce the observed anisotropies for $n=6$ in Fig. 4. All these indicators clearly demonstrate that gas phase cross sections are not suitable for the description of electron scattering in water cluster.

Better agreement between simulation and experiment than for pure condensed or pure gas phase scattering is obtained for the two intermediate cases (iii) and (iv) (full blue and full red lines in Fig. 4, respectively). For both cases, the values for $\beta_{\text {inp }}$ are in agreement with the corresponding monomer/small cluster data. ${ }^{20}$ Case (iv) appears to agree best with the experiment. For this case, the quasi-elastic cross sections are closer to the gas phase than to the condensed phase values (Fig. S1 in the ESI+), while the vibron and electronic cross sections are essentially those of the condensed phase (Figs. S2 and $\mathrm{S} 3$ in the ESI + ). It seems plausible that cluster scattering in the size range studied here can be treated as intermediate between condensed and gas phase scattering. Even for the largest clusters, many molecules are close to the surface. Those molecules do not experience the same environment as bulk molecules. The treatment of dielectric screening in cases (iii) and (iv) only affords a very simplistic description of the effect, and its physical meaning should not be overinterpreted. Moreover, dielectric screening is only one factor. Other factors include structural differences at or near the surface layer. Fewer or weaker hydrogen bonds do not only affect ionization energies but also change both the energy loss and the cross sections for phonon scattering, while the alignment of free OH-dipoles might modify the escape conditions for photoelectrons. None of these effects are 
accounted for by the treatment used here. Furthermore, previous studies have reported that at a cluster size of several hundred molecules water clusters can become (partly) crystalline ${ }^{59}$. To the best of our knowledge data on electron scattering (genuine and transport scattering) for crystalline water are not available so that we cannot clarify what the potential influence of crystalline structures on the experimental $\beta$ parameters could be.

Electron scattering might not be the only cause of the unexpectedly strong decrease of the experimental $\beta$ parameters with increasing cluster size (Fig. 4). Other potential factors include space charge effects, the presence of a small number of extremely large clusters, and related, carrier gas effects. High charge densities in the ionization volume, which can distort the trajectories of photoelectrons and result in too isotropic $\mathrm{PADs}^{28}$, can arise at high laser fluence and high sample density. In our experiments, we have made sure that the number of electrons per laser shot lies below the range where space charge effects play a role. However, there is another type of space charge effect that could arise from cluster ions formed in the cluster beam prior to laser ionization. Co-expansion of water with $\mathrm{He}$ or $\mathrm{Ne}$ carrier gas leads to high beam and thus high water cluster velocities. The kinetic energy of water clusters with $n \gtrsim 50$ and $n \gtrsim 200$, respectively, would be sufficient to ionize stainless steel surfaces (work function $\sim 4.4 \mathrm{eV}$ ) upon collision, ${ }^{60}$ for example at the repeller hole. We indeed observed a constant ion background signal due to this effects for expansions with $\mathrm{He}$ and Ne. By increasing the diameter of the repeller hole, however, we could remove this constant ion background and thus ascertain that collisional ionization of clusters had no effect on the measured $\beta$ parameters. This was also confirmed by the insensitivity of the $\beta$ parameters on the type of expansion (neat, $\mathrm{He}, \mathrm{Ne}$ ). It thus seems fairly safe to exclude space charges as a cause for the low cluster anisotropies we observe.

This leaves the presence of extremely large clusters as the only other potential contributing factor. Small amounts of water clusters with $n>10^{4}$ cannot be detected with the $\mathrm{Na}$ doping method because the detection efficiency of the MCP detector is insufficient for very heavy clusters. ${ }^{40}$ Note that such extremely large clusters are not accounted for either by using scaling laws for cluster sizing. The existence of a bimodal distribution with a second mode of giant clusters in low abundance was indeed detected in scattering experiments using a free-electron laser ${ }^{61}$, albeit for Xenon and not for water clusters. Since our photoelectron signal is proportional to the number of molecules in a cluster the experimental $\beta$ parameter (referred to as $\beta_{\exp }$ in the following) of a cluster distribution could be dominated by these extremely large clusters, even if their abundance were very low. The possible size range of such clusters, however, would be very limited by two considerations: Clusters with less than $n_{\text {large }}<10^{5}$ cannot reproduce $\beta_{\exp }$ and clusters with $n_{\text {large }}>10^{5}$ can either not reproduce $\beta_{\exp }$ and/or would show pronounced shadowing ${ }^{17}$ (see section 5 in the ESIt) in the calculated photoelectron images, which is not observed in the experimental images. This is exemplified by the data in Table 1 for the case of the $1 b_{1}$ orbital. It provides calculated $\beta$ parameters $\left(\beta_{\text {calc }}\right)$ for bimodal cluster size distributions which consist of a first mode of smaller clusters with a size of $\langle n\rangle=500$ and a second mode with a cluster sizes $n_{\text {large }}$ that varies between $5 \times 10^{4}$ and $5 \times$ $10^{5}$ water molecules. Furthermore, the percentages of small ( $\left.p_{\langle n\rangle}\right)$ and large $\left(p_{\text {large }}\right)$ clusters is varied. $\beta_{\text {calc }}$ is obtained from the summed simulated images calculated separately for the two modes $\left(n=\langle n\rangle\right.$ and $n=n_{\text {large, }}$ respectively; section $5 \mathrm{ESI}+$ ) using condensed phase scattering data (i.e. case (i)). The example data in Table 1 show that the experimental anisotropy parameter of $\beta_{\text {exp }}=0.34 \pm 0.06$ measured for $\langle n\rangle$

$\sim 500$ (Fig. 4) can only be reproduced within given uncertainties by setting $n_{\text {large }} \approx 10^{5}$. Smaller and larger values of $n_{\text {large }}$ are either not in agreement with $\beta_{\exp }$ or produce shadowing, which is not observed in the experimental spectra. Thus extremely large clusters would have to lie within an exceedingly narrow size range around $n_{\text {large }} \approx 10^{5}$ if their presence were to serve as an explanation for the observed size dependence of $\beta_{\exp }$ without invoking modified scattering cross sections for clusters compared with the condensed phase. The size and abundance of such clusters should depend noticeably on the cluster formation conditions with corresponding condition-dependent variations in $\beta_{\exp }$, which are, however, not observed. Instead we observe similar $\beta_{\exp }$ for similar $\langle n\rangle$ independent of the cluster formation conditions, viz. the type of expansion (neat, $\mathrm{He}, \mathrm{Ne}$ ), which speaks against a significant contribution to $\beta_{\text {exp }}$ from extremely large clusters. While this argument does not prove the absence of extremely large clusters, differences in the electron scattering properties of clusters compared with gas and condensed phase appear to offer the most plausible explanation for the size dependence of $\beta_{\exp }$ in Fig. 4.

Table 1: Example calculations for the anisotropy parameter for bimodal cluster distributions with a small clusters mode of size $\langle n\rangle$ and large clusters mode of size $n_{\text {large }} p_{\langle n\rangle}$ and $p_{\text {large }}$ are the percentages of the small and the large modes, respectively. $\beta_{\text {calc }}$ is calculated from the respective cluster sizes and their percentages using condensed phase scattering data (i. e. case (i)).

\begin{tabular}{ll|ll|rc}
\hline$\langle n\rangle$ & $p_{\langle n\rangle}$ & $n_{\text {large }}$ & $p_{\text {large }}$ & $\beta_{\text {calc }}$ & shadowing \\
\hline 500 & $90.0 \%$ & $5 \times 10^{4}$ & $10 \%$ & 0.41 & No \\
500 & $95.0 \%$ & $5 \times 10^{4}$ & $5.0 \%$ & 0.43 & No \\
\hline 500 & $90.0 \%$ & $10^{5}$ & $10 \%$ & 0.36 & No \\
500 & $95.0 \%$ & $10^{5}$ & $5.0 \%$ & 0.38 & No \\
\hline 500 & $99.5 \%$ & $2.5 \times 10^{5}$ & $0.5 \%$ & 0.42 & Yes \\
500 & $99.9 \%$ & $2.5 \times 10^{5}$ & $0.1 \%$ & 0.54 & No \\
\hline 500 & $99.5 \%$ & $5 \times 10^{5}$ & $0.5 \%$ & 0.37 & Yes \\
500 & $99.9 \%$ & $5 \times 10^{5}$ & $0.1 \%$ & 0.49 & No \\
\hline
\end{tabular}

\section{Conclusions}

The anisotropy parameter $\beta$ is a sensitive measure of lowenergy electron scattering in water clusters, especially for the elastic, phonon, and vibron scattering channels. With increasing cluster size, a surprisingly fast trend towards 
isotropic PADs is observed for $\left(\mathrm{H}_{2} \mathrm{O}\right)_{n}$ clusters with $\langle n\rangle<700$. The $\beta$ parameters of clusters with several hundred molecules already reach the value of liquid bulk water. We trace this behavior back to cluster specific electron scattering, even though the presence of a small fraction of extremely large clusters cannot be completely excluded as an additional factor.

Electron scattering simulations reveal that neither the gas nor the condensed phase scattering parameters appropriately describe electron scattering in $\left(\mathrm{H}_{2} \mathrm{O}\right)_{n}$ clusters with $\langle n\rangle<700$. Simulations with scattering cross sections that lie in between those of the gas and the condensed phase suggest that cluster scattering can be qualitatively understood as an intermediate case. In a very simple way, the partial inclusion of cluster-size independent dielectric screening can account for the different screening of molecules at a cluster's surface and in its volume. A more thorough interpretation of the experimental data will require the inclusion of other factors beyond dielectric screening. Experimental studies such as the present one provide the necessary data base and - we hope - the incentive for future theoretical work on low-energy electron scattering in water.

\section{Conflicts of interest}

There are no conflicts to declare.

\section{Acknowledgements}

We are very grateful to $\mathrm{Dr}$. Luca Poletto for his advice and for reviewing our design of the $\mathrm{HH}$ monochromator. We thank the group of Prof. Ursula Keller for advice on HHG, Dr. David Luckhaus for help with the scattering simulations, and David Stapfer and Markus Steger for technical support. Financial support was provided by the Swiss National Science Foundation under project nr. 200020_172472 and the ETH Zurich (project ETH-01 15-2 and ETH-FAST).

\section{References}

1. L. Sanche, Nature, 2009, 461, 358-359.

2. Q. B. Lu and L. Sanche, Phys. Rev. Lett., 2001, 87, 078501.

3. E. Alizadeh and L. Sanche, Chem. Rev., 2012, 112, 55785602.

4. H. Shinotsuka, B. Da, S. Tanuma, H. Yoshikawa, C. J. Powell and D. R. Penn, Surf. Interface Anal., 2017, 49, 238252.

5. S. Thürmer, R. Seidel, M. Faubel, W. Eberhardt, J. C. Hemminger, S. E. Bradforth and B. Winter, Phys. Rev. Lett., 2013, 111, 173005.

6. Y.-I. Suzuki, K. Nishizawa, N. Kurahashi and T. Suzuki, Physical Review E, 2014, 90, 010302.

$7 . \quad$ D. Luckhaus, Y. I. Yamamoto, T. Suzuki and R. Signorell, Sci. Adv., 2017, 3, e1603224.

8. G. Olivieri, K. M. Parry, C. J. Powell, D. J. Tobias and M. A Brown, J. Chem. Phys., 2016, 144, 154704.

9. R. L. Kurtz, N. Usuki, R. Stockbauer and T. E. Madey, J. Electron Spectrosc. Relat. Phenom., 1986, 40, 35-58.
10. J. C. Ashley, J. Electron Spectrosc. Relat. Phenom., 1988, 46, 199-214.

11. A. Akkerman and E. Akkerman, J. Appl. Phys., 1999, 86, 5809-5816.

12. M. Michaud and L. Sanche, Phys. Rev. A, 1987, 36, 46724683.

13. M. Michaud, A. Wen and L. Sanche, Radiat. Res., 2003, 159, 3-22.

14. D. Emfietzoglou and H. Nikjoo, Radiat. Res., 2005, 163, 98111.

15. C. J. Tung, T. C. Chao, H. W. Hsieh and W. T. Chan, Nucl. Instrum. Methods Phys. Res. Sect. B-Beam Interact. Mater. Atoms, 2007, 262, 231-239.

16. N. Ottosson, M. Faubel, S. E. Bradforth, P. Jungwirth and B. Winter, J. Electron Spectrosc. Relat. Phenom., 2010, 177, 60-70.

17. R. Signorell, M. Goldmann, B. L. Yoder, A. Bodi, E. Chasovskikh, L. Lang and D. Luckhaus, Chem. Phys. Lett., 2016, 658, 1-6.

18. C. Zhang, T. Andersson, M. Förstel, M. Mucke, T. Arion, M. Tchaplyguine, O. Björneholm and U. Hergenhahn, J. Chem. Phys., 2013, 138, 234306.

19. F. Blanco, A. Muñoz, D. Almeida, F. F. da Silva, P. LimãoVieira and G. García, Int. J. Mass Spectrom., 2014, 365, 287-294.

20. S. Hartweg, B. L. Yoder, G. A. Garcia, L. Nahon and R. Signorell, Phys. Rev. Lett., 2017, 118, 103402.

21. Y. Itikawa and N. Mason, J. Phys. Chem. Ref. Data, 2005, 34, 1-22.

22. J. Nishitani, C. W. West and T. Suzuki, Struct. Dyn., 2017, 4, 044014.

23. O. Björneholm, F. Federmann, S. Kakar and T. Möller, J. Chem. Phys., 1999, 111, 546-550.

24. G. Öhrwall, R. F. Fink, M. Tchaplyguine, L. Ojamäe, M. Lundwall, R. R. T. Marinho, A. N. de Brito, S. L. Sorensen, M. Gisselbrecht, R. Feifel, T. Rander, A. Lindblad, J. Schulz, L. J. Sæthre, N. Mårtensson, S. Svensson and $\mathrm{O}$. Björneholm, J. Chem. Phys., 2005, 123, 054310.

25. S. Barth, M. Ončák, V. Ulrich, M. Mucke, T. Lischke, P. Slavíček and U. Hergenhahn, J. Phys. Chem. A, 2009, 113, 13519-13527.

26. B. L. Yoder, A. H. C. West, B. Schläppi, E. Chasovskikh and R. Signorell, J. Chem. Phys., 2013, 138, 044202.

27. A. H. C. West, B. L. Yoder and R. Signorell, J. Phys. Chem. A, 2013, 117, 13326-13335.

28. R. Signorell, B. L. Yoder, A. H. C. West, J. J. Ferreiro and C.M. Saak, Chem. Sci., 2014, 5, 1283-1295.

29 A. H. C. West, B. L. Yoder, D. Luckhaus and R. Signorell, J. Phys. Chem. A, 2015, 119, 12376-12382.

30. A. O. Gunina and A. I. Krylov, J. Phys. Chem. A, 2016, 120, 9841-9856.

31. G. Öhrwall, M. Tchaplyguine, M. Gisselbrecht, M. Lundwall, R. Feifel, T. Rander, J. Schulz, R. R. T. Marinho, A. Lindgren, S. L. Sorensen, S. Svensson and $O$. Björneholm, J. Phys. B: At. Mol. Opt. Phys., 2003, 36, 3937-3949.

32. D. Rolles, Z. D. Pešić, H. Zhang, R. C. Bilodeau, J. D. Bozek and N. Berrah, XXV International Conference on Photonic, Electronic and Atomic Collisions, 2007, 88, 012003.

33. D. Rolles, H. Zhang, Z. D. Pešić, R. C. Bilodeau, A. Wills, E. Kukk, B. S. Rude, G. D. Ackerman, J. D. Bozek, R. Díez 
Muiño, F. J. García de Abajo and N. Berrah, Phys. Rev. A, 2007, 75, 031201.

34. D. Rolles, H. Zhang, Z. D. Pešić, J. D. Bozek and N. Berrah, Chem. Phys. Lett., 2009, 468, 148-152.

35. O. F. Hagena, Rev. Sci. Instrum., 1992, 63, 2374-2379.

36. C. Bobbert, S. Schütte, C. Steinbach and U. Buck, Eur. Phys. J. D, 2002, 19, 183-192.

37. S. Schütte and U. Buck, Int. J. Mass Spectrom., 2002, 220, 183-192.

38. B. L. Yoder, J. H. Litman, P. W. Forysinski, J. L. Corbett and R. Signorell, J. Phys. Chem. Lett., 2011, 2, 2623-2628.

39. J. H. Litman, B. L. Yoder, B. Schläppi and R. Signorell, Phys. Chem. Chem. Phys., 2013, 15, 940-949.

40. B. Schläppi, J. J. Ferreiro, J. H. Litman and R. Signorell, Int. J. Mass Spectrom., 2014, 372, 13-21.

41. R. M. Young and D. M. Neumark, Chem. Rev., 2012, 112, 5553-5577.

42. A. Sanov, Annu. Rev. Phys. Chem., 2014, 65, 341-363.

43. L. Ma, K. Majer, F. Chirot and B. von Issendorff, J. Chem. Phys., 2009, 131, 144303.

44. C. Bartels, C. Hock, J. Huwer, R. Kuhnen, J. Schwöbel and B. von Issendorff, Science, 2009, 323, 1323-1327.

45. J. J. Melko and A. W. Castleman, Jr., Phys. Chem. Chem. Phys., 2013, 15, 3173-3178.

46. Y.-I. Yamamoto, Y.-I. Suzuki, G. Tomasello, T. Horio, S. Karashima, R. Mitrić and T. Suzuki, Phys. Rev. Lett., 2014, 112, 187603.

47. A. Eppink and D. H. Parker, Rev. Sci. Instrum., 1997, 68, 3477-3484.

48. D. W. Chandler and P. L. Houston, J. Chem. Phys., 1987, 87, 1445-1447.

49. T. E. Gartmann, B. L. Yoder, E. Chasovskikh and R. Signorell, Chem. Phys. Lett., 2017, 683, 515-520.

50. U. Even, EPJ Tech. Instrum., 2015, 2, 22.

51. F. Frassetto, C. Cacho, C. A. Froud, I. C. E. Turcu, P. Villoresi, W. A. Bryan, E. Springate and L. Poletto, Opt. Express, 2011, 19, 19169-19181.

52. F. Frassetto, P. Miotti and L. Poletto, Photonics, 2014, 1, 442-454.

53. G. A. Garcia, L. Nahon and I. Powis, Rev. Sci. Instrum., 2004, 75, 4989-4996.

54. M. Ferray, A. L. Huillier, X. F. Li, L. A. Lompre, G. Mainfray and C. Manus, J. Phys. B: At. Mol. Opt. Phys., 1988, 21, L31.

55. T. E. Glover, R. W. Schoenlein, A. H. Chin and C. V. Shank, Phys. Rev. Lett., 1996, 76, 2468-2471.

56. B. Winter, R. Weber, W. Widdra, M. Dittmar, M. Faubel and I. V. Hertel, J. Phys. Chem. A, 2004, 108, 2625-2632.

57. L. Partanen, M.-H. Mikkela, M. Huttula, M. Tchaplyguine, C. Zhang, T. Andersson and O. Björneholm, J. Chem. Phys., 2013, 138, 044301.

58. O. Björneholm, F. Federmann, F. Fössing, T. Möller and P. Stampfli, J. Chem. Phys., 1996, 104, 1846-1854.

59. U. Buck, C. C. Pradzynski, T. Zeuch, J. M. Dieterich and B. Hartke, Phys. Chem. Chem. Phys., 2014, 16, 6859-6871.

60. U. Even, P. J. de Lange, H. T. Jonkman and J. Kommandeur, Phys. Rev. Lett., 1986, 56, 965-967.

61. D. Rupp, M. Adolph, L. Flückiger, T. Gorkhover, J. P. Müller, M. Müller, M. Sauppe, D. Wolter, S. Schorb, R. Treusch, C. Bostedt and T. Möller, J. Chem. Phys., 2014, 141, 044306. 